\title{
Cuidando Bem: um serious game sobre segurança do paciente para o ensino de enfermagem
}

\author{
Aline Natalia Domingues ${ }^{1}$, Catarine Santana Ohnuma ${ }^{2}$, Marcelo Lopes Lotufo ${ }^{2}$, \\ Breno Calixto Santos ${ }^{2}$, Rogério Augusto Bordini ${ }^{2}$, Joice Lee Otsuka ${ }^{2,3}$, Delano \\ Medeiros Beder ${ }^{2,3}$, Sílvia Helena Zem-Mascarenhas ${ }^{1 .}$ \\ ${ }^{1}$ Departamento de Enfermagem - Universidade Federal de São Carlos - São Carlos - SP \\ - Brasil \\ ${ }^{2}$ Laboratório de Objetos de Aprendizagem - Universidade Federal de São Carlos - São \\ Carlos - SP - Brasil \\ ${ }^{3}$ Departamento de Ciência da Computação - Universidade Federal de São Carlos - São \\ Carlos - SP -Brasil \\ \{aline.natalia, rogerio.bordini\}@sead.ufscar.br, \{catarina.ohn, \\ brenocalixtoas, marlolo1993\}@gmail.com, \{joice, silviazem\}@ufscar.br, \\ delanoddc.ufscar.br.
}

\begin{abstract}
Resumo. Este estudo tem como objetivo descrever um serious game desenvolvido como uma estratégia para o ensino de segurança do paciente. $O$ desenvolvimento do jogo percorreu até o momento as seguintes etapas: conceito, pré-produção, prototipação e produção de uma versão jogável (alpha). O jogo Cuidando Bem tem como objetivo educacional ensinar os protocolos de segurança do paciente lançados pelo Ministério da Saúde aos alunos do ensino profissionalizante e superior em enfermagem, de forma a contribuir à propagação do tema de segurança do paciente.
\end{abstract}

\section{Cenário de uso}

A assistência segura é um tema de relevância no contexto assistencial, tornando-se um assunto prioritário na área de saúde. O relatório "To err is human: building a safer health system" do Institute of Medicine (IOM) publicado em 1999 foi um marco importante para a segurança do paciente. Este estudo apontou que das 33,6 milhões de internações realizadas no ano de 1997, em hospitais dos EUA, por volta de 44.000 a 98.000 americanos morriam vítimas de iatrogenias médica [Kohn et al. 2001].

Em 2005, a Organização Mundial de Saúde (OMS) lançou a Aliança Mundial para a Segurança do Paciente e identificou seis metas para atuação, entre elas, o desenvolvimento de "Soluções para a Segurança do Paciente" [World Health Organization 2007].

Para a OMS, segurança do paciente é a redução do risco ou perigo desnecessário para o cuidado em saúde para um mínimo aceitável, conforme o conhecimento atual, os recursos disponíveis e o contexto da prestação do cuidado, em relação ao risco do não tratamento ou de outro tratamento [World Health Organization 2009]. 
Embora os princípios e conceitos de segurança do paciente são reconhecidos como essenciais na educação e formação do profissional de saúde, muitos profissionais e alunos continuam inseguros quanto à forma de integrar e aplicar o conhecimento de segurança do paciente na prática clínica [Leotsakos et al. 2014], [World Health Organization 2011].

A OMS (2011) lança o Guia Curricular Multiprofissional para Segurança do Paciente, com o intuito de acelerar a incorporação de ensino a segurança do paciente em currículos educacionais.

No Brasil, o Ministério da Saúde (MS), lança em 2013 o Programa Nacional de Segurança do Paciente (PNSP), que tem por objetivo geral contribuir para a qualificação do cuidado em saúde [Brasil 2013]. O PNSP traz como estratégia de implementação a articulação com o Ministério da Educação e o Conselho Nacional de Educação, para inclusão do tema segurança do paciente nos currículos dos cursos de formação em saúde de nível técnico, superior e pós-graduação [Brasil 2013].

O objetivo deste programa nacional está sendo o estabelecimento e a implementação de seis Protocolos de Segurança do Paciente, com foco nos problemas de maior incidência: Cirurgia segura; Prática de Higiene das mãos em serviços de saúde; Prevenção de úlceras por pressão; Prevenção de quedas em pacientes hospitalizados; Identificação do paciente; Segurança na prescrição e o uso de administração de medicamentos [Brasil 2013].

O ensino de segurança do paciente necessita de abordagens e modelos educacionais inovadores. Uma enorme variedade de métodos educacionais tem sido amplamente empregada nos currículos dos cursos de graduação para melhorar a formação dos estudantes, em favor da segurança do paciente [Gimenes e Cassiani 2014].

As novas tecnologias estão sendo cada vez mais utilizadas e integradas nos currículos da saúde, permitindo aos alunos o desenvolvimento de novas habilidades dentro de um ambiente seguro [Dariel et al. 2013]. O uso das tecnologias da informação e da comunicação (TIC) possibilita a inserção de novos recursos no contexto educacional, entre eles o serious game, como um potente instrumento pedagógico.

O serious game é caracterizado como um jogo educacional que promove a construção do conhecimento através de uma proposta pedagógica com conteúdos específicos, aliado a aspectos lúdicos que estimulam e motivam os processos de ensino aprendizagem [Bormann 2008].

Diante deste contexto, o presente trabalho apresenta o Cuidando Bem, um serious game que tem como objetivo educacional o ensino de segurança do paciente através dos protocolos lançados pelo MS, com abordagem de casos clínicos, tendo como público-alvo os alunos de cursos profissionalizantes (nível médio) e nível superior em enfermagem, uma vez que o contexto de segurança do paciente permeia todo aprendizado na concretização da assistência de enfermagem, contribuindo desta forma para o processo de ensino aprendizagem à promoção do tema segurança do paciente, através de uma estratégia educativa adicional. 
V Congresso Brasileiro de Informática na Educação (CBIE 2016)

Anais dos Workshops do V Congresso Brasileiro de Informática na Educação (CBIE 2016)

\section{Desenvolvimento}

O desenvolvimento do serious game é realizado com a colaboração de uma equipe do Laboratório de Objetos de Aprendizagem (LOA), da Secretaria Geral de Educação a Distância (SEaD) da Universidade Federal de são Carlos (UFSCar). O desenvolvimento do projeto iniciou-se no ano de 2014 e estende-se até o momento, tem sido realizado por estudantes de graduação e pós-graduação (Computação, Enfermagem, Letras, Imagem e Som, Música e Psicologia), sob a orientação e coordenação de docentes das áreas de Computação e Enfermagem.

Por se tratar de uma equipe interdisciplinar, o serious game foi desenvolvido segundo uma abordagem colaborativa e equilibrada: enquanto os programadores e os ilustradores se empenham na elaboração de uma mecânica divertida e funcional ao jogador, os docentes e alunos responsáveis pelo conteúdo educacional construíram um sistema de aprendizagem significativo e didático, de forma que não fosse ofuscada pela narrativa ou outros elementos, sendo um dos maiores desafios do projeto. É nesse contexto que o LOA, vem desenvolvendo suas pesquisas concentradas na utilização dos games como objetos capazes de proporcionar um aprendizado lúdico ao estudante/jogador.

O Cuidando Bem é um serious game, em 2D, point-click e desenvolvido para multiplataforma. Optou-se pelo uso da linguagem de programação HTML 5 (Hypertext Markup Language, versão 5) por suportar múltiplas plataformas de hardware e software, considerando que é uma linguagem multiplataforma e a única exigência para sua execução é a presença de navegadores que estejam em conformidade com a especificação. Além disso, foi utilizado o JavaScript, uma linguagem interpretada que normalmente é usada para executar scripts do lado do usuário apenas, sem necessidade de comunicação com o servidor. Uma das funcionalidades é, por exemplo, a detecção de teclas pressionadas pelo usuário, algo que o HTML sozinho não conseguiria detectar, logo torna-se possível controlar, por exemplo, a movimentação de um objeto em tela usando as teclas do teclado. Por esse script rodar apenas do lado do jogador, a ação torna-se mais responsiva.

Os estudos de Novak (2010) e Schell (2011) se complementam no escopo de game design por isso, foram escolhidos para o delineamento metodológico do serious game e auxílio à construção dos documentos de design necessários. $\mathrm{O}$ roteiro utilizado para elaboração dos documentos foi desenvolvido através da equipe interdisciplinar.

O desenvolvimento do serious game ocorre através de ciclos iterativos de testes, análises, refinamentos do design/protótipo. Até o momento o serious game percorreu as seguintes etapas: conceito, pré-produção (documentação do jogo), prototipação e a produção de um jogo educacional em versão alpha.

O Cuidando Bem já passou por uma avaliação inicial do protótipo, com estudantes do ensino profissionalizante de enfermagem, que teve como objetivo avaliar esta tecnologia na perspectiva dos estudantes de enfermagem, como etapa importante para a mensuração da jogabilidade e impacto do mesmo para implementação futura no processo de ensino e aprendizagem. Os resultados dessa avaliação encontra-se em maior detalhe no presente trabalho apresentado e inserido nos anais do XX Congresso Internacional de Informática Educativa/TISE 2015 [Domingues et al. 2015]. 
V Congresso Brasileiro de Informática na Educação (CBIE 2016)

Anais dos Workshops do V Congresso Brasileiro de Informática na Educação (CBIE 2016)

\section{Apresentação do Software}

O Cuidando Bem foi produzido até sua versão alpha na qual suas propriedades e funcionalidades já foram implementadas. No entanto, esta versão ainda contém alguns erros, tais como problemas de balanceamento e interface [Benthke 2003].

Esta versão alpha do Cuidando Bem é composta por um total de dez fases, constituídas por níveis de dificuldades gradativos através da apresentação dos protocolos de segurança do paciente. O jogo traz a história de um profissional de enfermagem que foi recém-contratado por um hospital e está em período de três meses de experiência.

Desta forma, o jogador inicia seu primeiro dia da sua experiência no hospital. Após um encontro com seu enfermeiro responsável que lhe mostra o funcionamento básico do hospital e suas tarefas. A cada fase o jogador vai ter que trabalhar em um caso clínico específico desenvolvido para o jogador ter experiência e compreender a aplicação de todos os protocolos de segurança do paciente. Conforme o jogador for avançando nos jogos, esses casos ficarão mais complexos e exigirão maior conhecimento e atenção do jogador. O jogo é composto por dez fases, elevando gradualmente o nível dificuldade, as três primeiras fases aparecem maior número de intervenções, para que o jogador realize as ações corretas, através do personagem mentor.

Utilizando um sistema point-click o jogador deverá utilizar os objetos no cenário, ações e interações com outros personagens para avançar nas fases. Sempre que o jogador tomar as decisões ele receberá um feedback. Dependendo do número de decisões corretas e erradas tomadas o jogador poderá ser contratado no final do período de experiência. Os casos clínicos são apresentados através de simulações do trabalho da enfermagem frente à assistência ao paciente. Os jogos educacionais e a simulação digital contribuem para que o conhecimento, adquirido em experiências virtuais, seja aplicado nas situações reais [Teixeira e Félix 2011].

A versão do jogo para acesso encontra-se disponível no website do $\mathrm{LOA}^{1}$ e através do Github. O Github ${ }^{2}$ é um Serviço de Web Hosting compartilhado para projetos que usam o controle de versionamento Git, o mesmo, possui funcionalidades de uma rede social, além de permitir visualizar como os desenvolvedores trabalham as versões de seus repositórios.

O jogo desenvolvido apresenta uma licença do tipo Creative Commons, pois trata-se de um recurso educacional aberto voltado para o uso e reuso da comunidade. Os pacotes de instalação do jogo, bem como os códigos fontes, serão compartilhados com a comunidade de software livre. O serious game, bem como todos os seus componentes, ficarão disponíveis para a comunidade no Repositório Digital Livre Saber ${ }^{3}$, repositório digital de acesso livre da SEaD/UFSCar, onde está sendo organizado um acervo de objetos educacionais multimídia produzidos como recursos educacionais abertos. A seguir são apresentadas algumas telas do serious game Cuidando Bem.

\footnotetext{
${ }^{1}$ http://www.loa.sead.ufscar.br/cuidandobem.php

${ }^{2}$ http://loa-sead.github.io/cuidando-bem/

${ }^{3}$ livresaber.sead.ufscar.br
} 
V Congresso Brasileiro de Informática na Educação (CBIE 2016)

Anais dos Workshops do V Congresso Brasileiro de Informática na Educação (CBIE 2016)

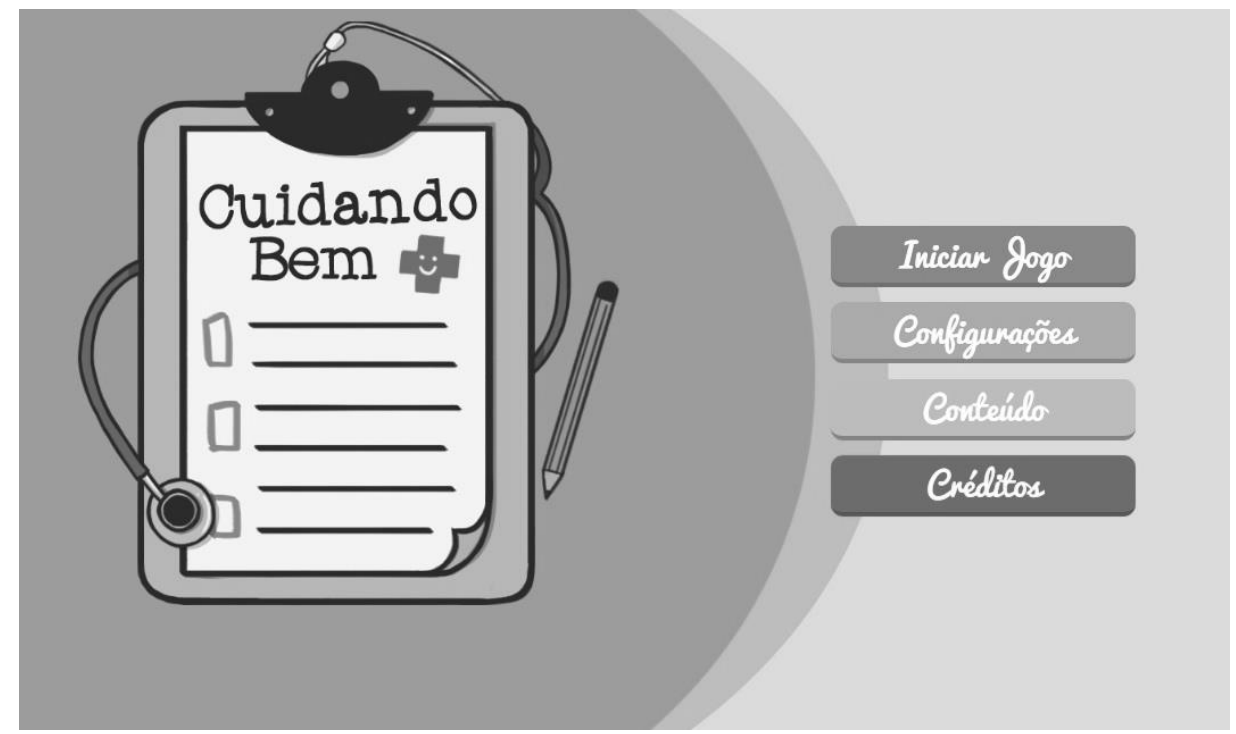

Figura 1. Tela inicial do Cuidando Bem.

O jogo apresenta uma interface inicial na qual é possível selecionar iniciar um jogo novo ou carregar um jogo em andamento previamente salvo. Além disso, apresenta menus de configurações, conteúdo e créditos. O menu de conteúdo nesta versão não está disponível, pois estão em fase de desenvolvimento final para inserção no jogo, nele o jogador terá informações da mecânico do jogo, assim como todo conteúdo dos protocolos de segurança do paciente e um dicionário de termos e siglas que aparecem durante ao jogo.

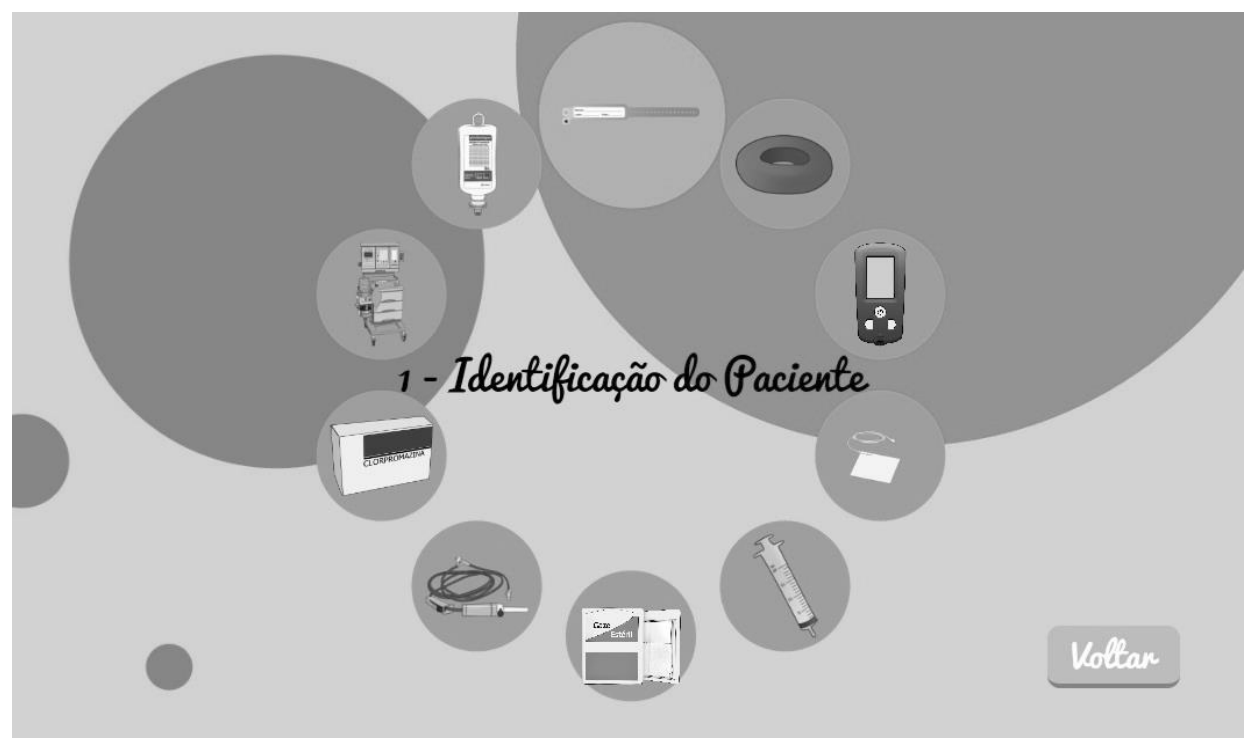

Figura 2.Tela de exibição com todas as fases desbloqueadas.

As ações dos jogo e suas interações são realizadas através do point-click, e são pontuadas através de um score, no qual é apresentado ao jogador após o término de cada 
V Congresso Brasileiro de Informática na Educação (CBIE 2016)

Anais dos Workshops do V Congresso Brasileiro de Informática na Educação (CBIE 2016)

fase. Os cenários apresentados no jogo para exploração do jogador são recepção, corredor, enfermarias (feminina e masculina), posto de enfermagem, farmácia e centro cirúrgico.

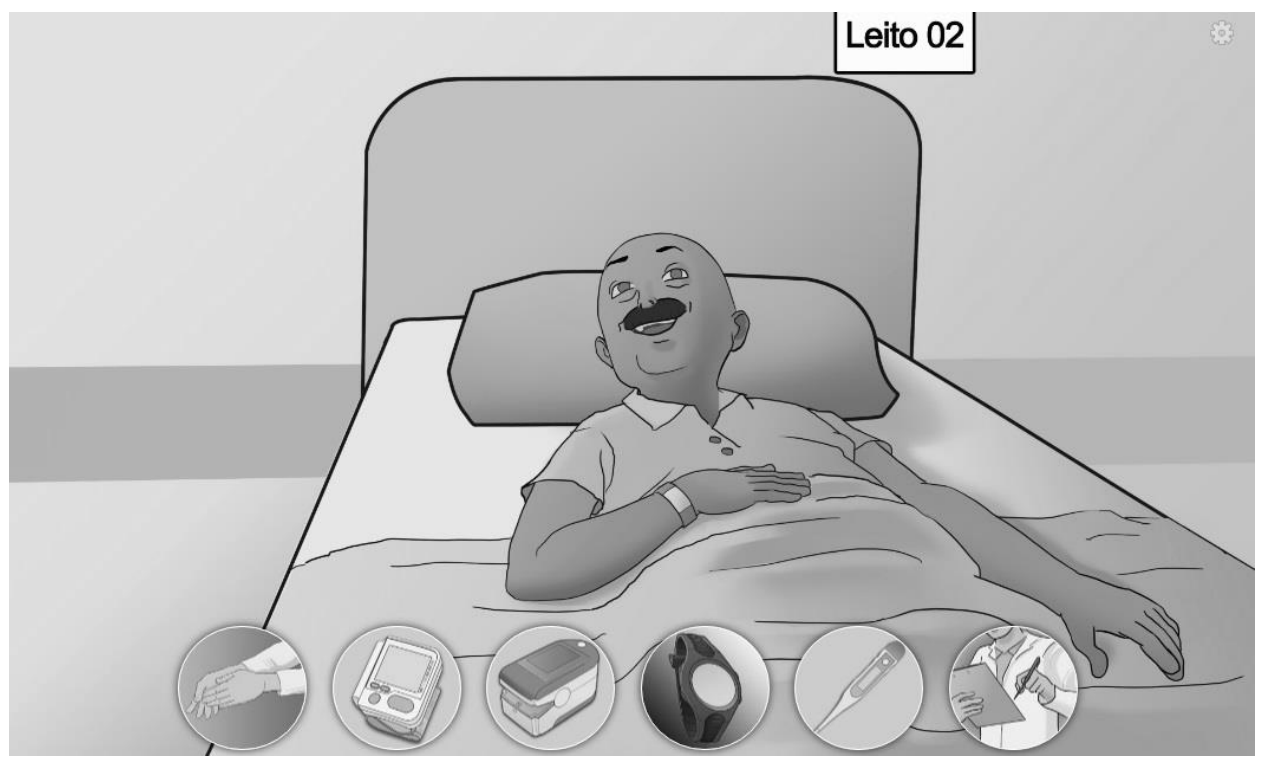

Figura 3. Exemplo de tela de interatividade de objetos relacionados às ações.

Além disso, o jogo permite realizar outras ações mais complexas como o cálculo do gotejamento de soro e a aferição da frequência respiratória no tempo de um minuto, através de animações. Também é possível que o jogador digite em campos validados as informações pertinentes para realização do caso clínico, como preenchimento da pulseira de identificação, prontuário do paciente e ficha de medicação, visando que a anotação e registro são um dos passos fundamentais para a segurança do paciente.

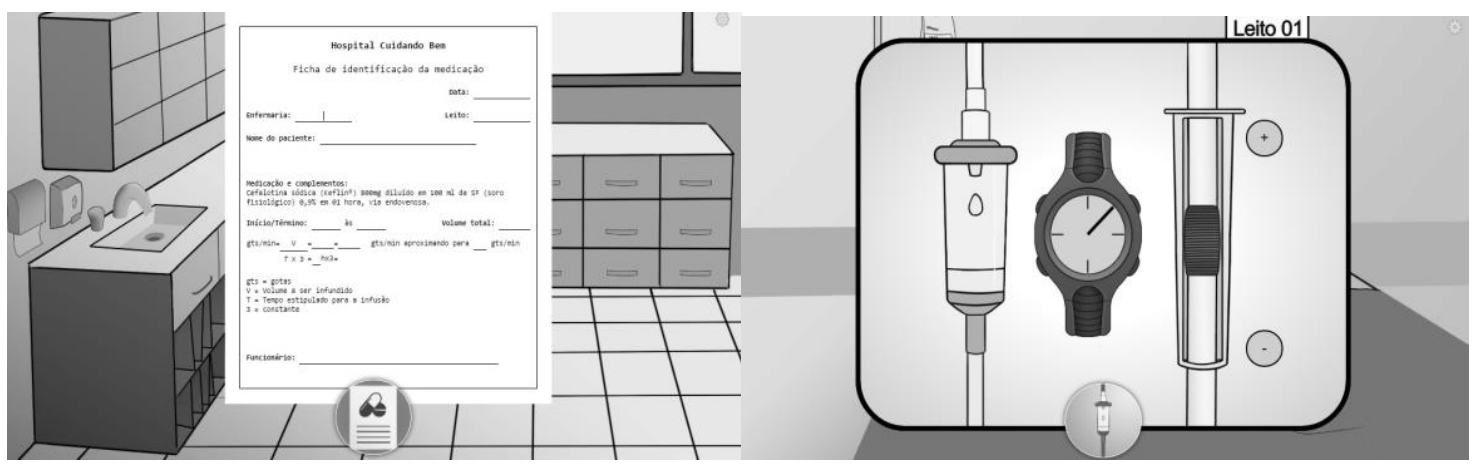

Figura 4. Tela da ficha de medicação e gotejamentos de soro.

É válido ressaltar que em nenhum momento do jogo, o jogador realizará ações que causem dano ao paciente (exemplo administrar medicação errada, óbito do paciente), pensando na questão da prevenção de iatrogenias que envolve a cultura de segurança do paciente, e com o intuito de não impactar o estudante negativamente na assistência de enfermagem. O jogador vence e é contratado no hospital Cuidando Bem se atingir um score igual ou acima de $75 \%$ em todas as fases. 
V Congresso Brasileiro de Informática na Educação (CBIE 2016)

Anais dos Workshops do V Congresso Brasileiro de Informática na Educação (CBIE 2016)

\section{Considerações finais}

$\mathrm{Na}$ enfermagem, observa-se a necessidade de inovações no processo de ensinoaprendizagem que estimulem o conhecimento e o raciocínio clínico. Evidencia-se que o uso de recursos educacionais permite a realização de processo de ensino mais criativos e facilita uma aprendizagem mais ativa por parte dos alunos.

O serious game Cuidando Bem apresentado neste trabalho, tem como objetivo ser uma estratégia para o processo de ensino aprendizagem de segurança do paciente no ensino de enfermagem. Desde a sua concepção e implementação, houve enorme preocupação em aliar os objetivos educacionais à jogabilidade, envolvendo uma equipe interdisciplinar no desenvolvimento do jogo.

O jogo também está sendo projetado para ser acessível para pessoas com deficiência visual e baixa mobilidade, buscando seguir princípios de design universal que reduzam barreiras de acesso e uso [Domingues et al. 2014].

O protótipo inicial do serious game já foi testado pelo público-alvo dos alunos do ensino profissionalizante de enfermagem, no qual se obteve resultados positivos na utilização do mesmo como um recurso educacional de apoio ao ensino de segurança do paciente, porém evidencia-se a necessidade de realizar mais testes e avaliações do software [Domingues et al. 2015].

Devido a esta lacuna, como trabalhos futuros pretende-se realizar testes de avaliação por especialistas da área de saúde e de computação, no qual serão aplicados questionários semiestruturados com base no método de avaliação de jogos educacionais UsaECG [Mohamed-Omar, Yusoff e Jaafar 2012] e também será avaliado por alunos de curso profissionalizante e de nível superior de enfermagem a sua satisfação com os conteúdos e a jogabilidade do jogo proposto a partir do método EgameFlow [Fu, Su e $\mathrm{Yu}$ 2009]. Os resultados destas avaliações subsidiarão as mudanças e melhorias na versão final do jogo.

Espera-se que os resultados deste estudo forneçam evidências que sustentem a importância de estratégias educacionais inovadoras no processo de formação dos alunos de cursos profissionalizantes e de nível superior na área da enfermagem.

\section{Referências}

Benthke, E. (2003) Game design document, Game development and Production. Wordware Publishing, Inc. Plano, Texas, p. 101-127.

Bormann, M. et al. (2008) "Spielend lernen! Spielend lernen? Eine empirische annäherung an die möglichkeit einer synthese von spielen und lernen”. In: U. Lucke, M.C. Kindsmüller, S. Fischer, M. Herczeg, S. Seehusen (Eds.), Tagungsband der Konferenz DelFI 2008, in Lübeck, Oldenbourg, Munich, p. 339-343.

Brasil. Ministério da Saúde. (2013) Portaria n.529, de $1^{\circ}$ de abril de 2013. Diário Oficial da República Federativa do Brasil, Brasília (DF).

Dariel O. J. P. et al. (2013) Developing the Serious Games potential in nursing education. Nurse Education Today, v.33, p. 1569-1575. 
V Congresso Brasileiro de Informática na Educação (CBIE 2016)

Anais dos Workshops do V Congresso Brasileiro de Informática na Educação (CBIE 2016)

Domingues, A. N. et al. (2014) Uso de protótipo em papel no design de um jogo educacional acessível. In: Simpósio Brasileiro de Jogos e Entretenimento Digital XII SBGames, 2014, Porto Alegre, RS. SBGames, 2014. http://www.sbgames.org/sbgames2014/files/papers/art_design/full/A\&D_Full_Uso\% 20de\%20prototipo\%20em\%20papel.pdf. May

Domingues, A. N. et al. (2015) Jogo educacional sobre segurança do paciente: avaliação de estudantes de enfermagem. In: XX Congresso Internacional de Informática em Educativa (TISE 2015), 2015, Santiago, Chile. Nuevas Ideas en Informática Educativa TISE 2015 , 2015. $\quad$ p. http://www.tise.cl/volumen11/TISE2015/684-688.pdf, May.

Fu, F. L., Su, R. C., e Yu, S. C. (2009) EGameFlow: A scale to measure learners' enjoyment of e-learning games. Computers \& Education. 52(1):101-112.

Gimenes, F. R. E. e Cassiani, S. H. B. (2014) "Capítulo II: Segurança e qualidade dos cuidados”. In: MARTINS, J. C. A.; MAZZO, A.; MENDES, I. A. C. Simulação no ensino de enfermagem. Ribeirão Preto: SOBRACEn, p. 39-51.

Kohn, L. T. et al. (2001) To Error is human: building a safer health system. Washington: Committee on Quality of Health Care in America, National Academy of Institute of Medicine.

Leotsakos, A. et al. (2014) Educating future leaders in patient safety. J Multidiscip Health. 19; 7:381-8.

Mohamed-Omar, H., Yusoff, R., Jaafar, A. (2012) "Quantitive analysis in a heuristic evaluation for usability of Educational Computer Game (UsaECG)," Information Retrieval \& Knowledge Management (CAMP), 2012 International Conference on, vol., no., pp.187,192, 13-15.

Novak, J. (2010) Game development essentials:an introduction. Ed. Cengage Learning.

SCHELL J. (2011) A arte de game design: O livro original. Rio de Janeiro, Brasil: Elsevier.

Teixeira, I. N. D. e Félix, J. V. C. (2011) Simulação como estratégia de ensino em enfermagem: revisão de literatura. Interface (Botucatu), v. 15, n. 39, p. 1173-1184.

World Health Organization. (2007) "Patient Safety Solutions Preamble", http://www.who.int/patientsafety/solutions/patientsafety/Preamble.pdf, May.

World Health Organization. (2009) "The Conceptual Framework for the International Classification for Patient Safety". Versio 1.1. Final Technical Report. Chapter 3. The International Classification for Patient Safety. Key Concepts and Preferred Terms. Geneva.

World Health Organization. (2011) Patient Safety Curriculum Guide for Medical Schools, Evaluation Study. Geneva. 